\title{
ASSOCIATION OF HYPERTENSION WITH TYPE 2 DIABETES MELLITUS IN ADULT FEMALE
}

\author{
NAHID YEASMIN ${ }^{1}$, SAYEEDA MAHMUDA ${ }^{2}$, SHAMIMA AKTHER, ${ }^{3}$ SHARMIN NAHAR ${ }^{4}$, ZAKIA SULTANA $^{5}$, \\ AFRUZA KHANAM ${ }^{6}$
}

\begin{abstract}
Background: Diabetes mellitus is one of the most widespread endocrine disorder in female and its complications are increasing all over the world, leading to life threatening medical problems like cardiovascular diseases, stroke and end stage renal diseases. An association between hypertension and diabetes mellitus has been identified.
\end{abstract}

Objectives: The study was carried out to observe the association of hypertension with diabetes mellitus in adult female subjects.

Methods : This cross sectional study was conducted in the Department of Physiology, Dhaka Medical College, Dhaka, during the period of January 2011 to December 2011. A total number of one hundred female subjects were selected with age ranging from 30 to50 years. Among them 50 diabetic female subjects were included from out-patient department of Endocrinology, Dhaka Medical College Hospital, Dhaka as study group(B) and 50 apparently healthy female were taken as control group(A) for comparison. Fasting serum insulin level was measured by ELISA method in the Department of Laboratory of National Institute of ENT, Dhaka and fasting blood glucose was estimated by glucose oxidase method in Department of Dhaka Medical College in both groups. Systolic blood pressure and diastolic blood pressure of all participants were recorded by standard method. Data was analyzed by Unpaired Student's- test and Pearson's correlation co-efficient (r) test as applicable.

Results: The value of fasting serum insulin level was significantly higher in diabetic subjects than those of non-diabetic subjects. Fasting blood glucose level was significantly higher diabetic patient in comparison to those of non-diabetic subjects. In diabetic subjects fasting serum insulin and fasting blood glucose levels were higher and showed positive correlation with systolic and diastolic blood pressure.

Conclusion: Present study revealed that systolic and diastolic blood pressure has positive relationship with fasting serum insulin and fasting blood glucose levels.

Key words: Fasting serum insulin, fasting blood glucose, hypertension, diabetes mellitus.

Received: 01 July 2017

Accepted: 28 November 2017

DOI: http://dx.doi.org/10.3329/bjmed.v29i1.35402

\section{Introduction}

Diabetes mellitus (DM) is a metabolic disorder characterized by chronic hyperglycemia with disturbances of carbohydrate, fat and protein metabolism resulting from defects in insulin secretion and / or insulin action. ${ }^{1}$ Diabetes mellitus is one of the common non-communicable diseases and it is one of the most challenging health problems in the $21^{\text {st }}$ century. There are two general types of diabetes mellitus, one is type 1 or insulin dependent DM (IDDM) and other is type 2 or non-insulin dependent DM. Among them, type 2 diabetes mellitus is more common and about 90 to $95 \%$ of all cases of diabetes mellitus. ${ }^{2}$

1. Assistant Professor, Department of Physiology, Dhaka Medical College, Dhaka

2. Associate Professor (CC), Department of Pathology ,National Institute of Ophthalmology \& Hospital, Sher-E-Bangla Nagar, Dhaka.

3. Assistant Professor, Department of Physiology, Dhaka Medical College, Dhaka

4. Lecturer, Department of Physiology, Dhaka Medical College, Dhaka

5. Associate Professor, Department of Physiology, Dhaka Dental College.

6. Assistant Professor, Department of Physiology, Marks Medical College.

Address of Correspondence: Dr. Nahid Yeasmin, Assistant Professor, Department of Physiology, Dhaka Medical College, Dhaka

Bangladesh J Medicine 2018; 29 : 7-12 
According to International Diabetic Federation (IDF), about 24.4 million diabetic people in USA, 65.1 million in India and 5.1million in Bangladesh. This number is gradually increasing day by day will rise to 592 million by the year 2035 representing about $8.8 \%$ of the world population. By the year 2035, the number of diabetic people will be about 29.7 million in USA, 109.2 million in India and 16.8 million in Bangladesh. ${ }^{3}$

Insulin resistance represents the earliest detectable abnormality in of type 2 diabetes mellitus and is one of the major underlying mechanisms of hypertension and cardiovascular diseases. ${ }^{4}$ Insulin is the most potent anabolic hormone which is secreted by beta cells of pancreatic islets of Langerhans in response to increased circulating level of glucose and amino acid after a meal. Insulin regulated glucose homeostasis by increasing the rate of glucose uptake into skeletal muscle and adipose tissue and reducing hepatic glucose output. ${ }^{5}$

Insulin resistance refers to the reduction in insulin mediated glucose uptake in insulin-sensitive tissue specifically in skeletal muscle. As a compensatory response, hyperinsulinemia ensues to maintain normal blood glucose level. 6

A number of studies have shown the relationship between insulin resistances in type- 2 diabetes mellitus and hypertension. They found that type- 2 diabetes mellitus patients with insulin resistance have more chance for development of hypertension. ${ }^{7-}$ 12. They suggested that the influence of insulin on blood pressure may be altered in insulin resistance. The insulin induced vasodilation is impaired due to defect in PI3 (phosphoinositide-3) kinase signaling in insulin resistance. Moreover, recent data suggested that insulin induced enhancement of renal sodium reabsorption is preserved or even enhanced in insulin resistance. 13

The prevalence of hypertension is increasing throughout the world. It is known as "the silent killer", it may exit for prolonged periods without symptoms and may manifest only after causing serious complications ${ }^{14}$.Hypertension is a recognized modifiable risk factor of cardiovascular disease, stroke and end stage renal disease. The prevalence of hypertension has increased in women than man. ${ }^{15}$ According to global estimates, in 2000, 972 million adult had hypertension and it is predicted to rise by to $60 \%$ to a total 1.56 billion by $2025^{16}$ Insulin resistances is a risk factor for development of hypertension and hypertension is approximately twice as prevalent in the type 2 diabetes mellitus. ${ }^{17}$
A study was done in USA and they found that up to $75 \%$ of adult with diabetes mellitus have hypertension and patient with hypertension also show evidence of insulin resistance. Thus hypertension and diabetes are common intertwined condition that shares a significant overlapping risk factors and complications. ${ }^{18}$ Hypertension and diabetes affects approximately 74.5 million and 23.6 million adult in the United Sates respectively and approximately $75 \%$ of patient with DM have concomitant hypertension. The economic impact of hypertension and diabetes mellitus are enormous burden on society with estimated annual costs of 174 billion for diabetic care and 76.6 billion for hypertension related problems There is a significant amount of overlap between the complications of diabetes mellitus and hypertension. These complications can divided into macrovascular and microvascular complications including coronary artery disease, myocardial infarction, congestive cardiac failure, stroke, peripheral vascular diseases and end stage renal diseases. ${ }^{19}$

Insulin resistance and its consequence of hyperinsulinemia have been recognized as a most important risk factor for developing hypertension. Several epidemiological studies from different populations have been reported a significant association between insulin resistance and hypertension. They suggested that more than 20 million American have type 2 diabetes caused by insulin resistance and 25 to $40 \%$ of entire population have earlier stages of insulin resistance.

A number of studies have shown the insulin resistance in DM increases the hypertension. They also reported that by improving insulin sensitivity through diet and regular physical exercise has been associated with reduction in blood pressure. ${ }^{18}, 20$,

The link between insulin resistance and hypertension is neuroendocrine mechanisms including excess sodium retention, increasing sympathetic activities, renin-angiotensin-aldosterone system (RAAS) activation, alteration of trans membrane ion transport, hypertrophy of resistance vessels, endothelial dysfunction and oxidative stress. ${ }^{21,22}$

Some studies have been done on this regard in abroad but no published data has yet been available on this aspect in our country. Therefore, the present study has been designed to observe the association of hypertension with type 2 diabetes mellitus in adult female. Better understanding of these metabolic changes with insulin resistance in type 2 diabetes mellitus patient will help in the detection of women at risk for future cardiovascular diseases and renal diseases. Treatment and preventive measure can 
reduce the risk of developing cardiovascular diseases and thus reduce burden on our health budget.

\section{Methods}

This cross sectional study was conducted in the Department of Physiology, Dhaka Medical College, Dhaka, during the period of January 2011 to December 2011. Fifty female of 30-50 years of age range with diabetes mellitus in this study as study (B) group. They were selected from out- patient Department of Endocrinology of Dhaka Medical College, Hospital; Dhaka. Age matched fifty apparently healthy females studied as control (A) group for comparison. Subjects having history of heart, liver, kidney diseases, endocrine disorders and women taking hormone replacement therapy, steroid, alcohol user, and smoker were excluded from the study. After selection of the subjects, the objectives, nature, purpose and benefit of the study were explained to the subjects in details. Ethical permission was taken from ethical committee of Dhaka Medical College. After selection of subjects, the objectives, nature, purpose and benefit of the study were explained to the subjects in details. Written informed consents were taken from the participants. The subjects were advised to attend the laboratory in the Department of Physiology of Dhaka Medical College, Dhaka between 8AM to 10AM. Detailed personal history, medical history, drugs history, family history and all the clinical examinations were done. Then with all aseptic precautions $5 \mathrm{ml}$ of venous blood was drawn from antecubital vein by disposable plastic syringe. Blood was allowed to clot and then centrifuged at rate of $3000 \mathrm{rpm}$ and supernatant clear serum was separated Serum was taken in a eppendrof tube and was preserved in refrigerator in Department of Physiology of Dhaka Medical College, Dhaka. Fasting serum insulin level was assessed by Enzyme Linked Immunosorbent Assay (ELISA) and fasting blood

glucose level was estimated by glucose oxidase method in the laboratory of Department of Physiology. The blood pressure of the subjects was measured after 5 minutes rest with a sphygmomanometer using an appropriate cuff size in the right arm. Hypertension was defined according to the Joint National Committee (JNC) VII criteria. ${ }^{22}$ Statistical analysis was done by Unpaired Student's t- test. Correlation was analyzed by Pearson's correlation coefficient $(\mathrm{r})$ test. $\mathrm{P}$ value $<0.05$ was taken as of significance.

\section{Results}

The value of mean serum insulin of diabetic subject was significantly higher than that of controls. The level of mean blood glucose was also higher in diabetic adult female than that of controsl and result was statistically significant. Table-I

Mean values of systolic and diastolic blood pressure were higher in diabetic adult female than that of controls and results were statistically significant. Table-II.

Again, distribution of parameters were observed in diabetic subjects and $17.3 \%$ had serum insulin level within normal level whereas, $82.7 \%$ had above normal level (i.e $>25 \mathrm{uU} / \mathrm{ml}$ ). Again, $21.7 \%$ of diabetic subjects had blood glucose level within normal level whereas, $78.3 \%$ had above normal level. Table III

Serum fasting insulin level showed positive correlation with systolic blood pressure and result was statistically significant. Again serum insulin level showed positive correlation with diastolic blood pressure diabetic subjects but result was statistically non significant.Table- IV and Figure-1,2. Again, fasting blood glucose level also showed positive correlation with systolic and diastolic blood pressure in diabetic subjects result was statistically non significant . Table-V and Figure 3 and 4

Table I

Age, Fasting serum insulin (FSI) and Fasting blood glucose (FBG) in both groups

\begin{tabular}{ccccc}
\hline Groups & $\mathrm{n}$ & $\begin{array}{c}\text { Age (years) } \\
(\text { Mean } \pm \text { SD) }\end{array}$ & $\begin{array}{c}\text { FSI(uIU/ml) } \\
(\text { Mean } \pm \text { SD) }\end{array}$ & $\begin{array}{c}\text { FBG(mg/dl) } \\
(\mathrm{Mean} \pm \mathrm{SD})\end{array}$ \\
\hline A & 50 & $45.60 . \pm 4.66$ & $9.5 .03 \pm .3 .07$ & $90.10 \pm .27 .457$ \\
B & 50 & $48.0 \pm 4.75$ & $20.13 \pm .8 .41$ & $120.14 \pm 30.17$ \\
Statistical analysis & Age & FSI & FBG \\
Groups & & (p value) & (p value) & $0.003^{* *}$ \\
A vs B & & $0.113^{\text {ns }}$ & $0.001^{* * *}$ & (pvalue) \\
\hline
\end{tabular}

Unpaired Student's't' test was performed to compare between groups. The test of significance was calculated and $\mathrm{p}$ values $<0.05$ was accepted as level of significance.

\begin{tabular}{|c|c|c|c|}
\hline Group A & Healthy adult female & $\mathrm{n}$ & Number of subjects \\
\hline Group B & Diabetic adult female & $\mathrm{ns}$ & Not significant \\
\hline
\end{tabular}

$* / * * / * * \quad=$ Significant

(1) 
Table-II

Systolic and Diastolic blood pressure in both groups

\begin{tabular}{lcc}
\hline Parameters & $\begin{array}{c}\text { Group A } \\
\text { (Control) }\end{array}$ & $\begin{array}{c}\text { Group B } \\
\text { (Diabetic patients) }\end{array}$ \\
\hline Systolic blood & $118.00 \pm 13.68$ & $140.58 \pm 9.44$. \\
pressure (mmHg) & & \\
Diastolic blood & $70.67 \pm 8.61$ & $85.67 \pm 10.61$ \\
pressure (mmHg) & & \\
\hline
\end{tabular}

\section{Results are expressed as mean \pm SD}

Unpaired Student's ' $t$ ' test was performed to compare between groups. The test of significance was calculated and $\mathrm{p}$ values $<0.05$ was accepted as level of significance.

Group A : Adult healthy female

Group B : Diabetic adult female

Table-III

Distribution of the subjects by the study parameters in diabetic female

\begin{tabular}{lcc}
\hline Parameters & \multicolumn{2}{c}{ Group B (n=50) } \\
& No. & $(\%)$ \\
\hline Fasting serum insulin (uUL/ml) & & \\
$<25$ & 9 & $(17.3)$ \\
$>25$ & 41 & $(82.7)$ \\
Fasting blood glucose (mg/dl) & & \\
$<126$ & 14 & $(28.0)$ \\
$>126$ & 36 & $(72.0)-$ \\
\hline
\end{tabular}

$\mathrm{N}=$ Number of subjects

Group B: Study group (adult diabetic female)

Table-IV

Correlation of fasting serum insulin level with SBP and $D B P$ with in diabetic adult female

Parameters Group B $(n=50)$

\begin{tabular}{lcc} 
& $\mathrm{r}$ & $\mathrm{p}$ \\
\hline Systolic blood pressure (SBP) & +0.279 & 0.014 \\
Diastolic blood pressure (DBP) & +0.138 & 0.119 \\
\hline
\end{tabular}

Pearson's correlation coefficient (r) test was performed to compare relationship between parameters. The test of significance was calculated and $\mathrm{p}$ value $<0.05$ was accepted as level of significance.
Table V

Correlation of blood glucose level with SBP and DBP in diabetic adult female

\begin{tabular}{lcc}
\hline Parameters & \multicolumn{2}{c}{ Group B (n=50) } \\
& $\mathrm{r}$ & $\mathrm{p}$ \\
\hline Systolic blood pressure (SBP) & +0.098 & 0.422 \\
Diastolic blood pressure (DBP) & +0.004 & 0.917 \\
\hline
\end{tabular}

Pearson's correlation coefficient (r) test was performed to compare relationship between parameters. The test of significance was calculated and $p$ value $<0.05$ was accepted as level of Significance.

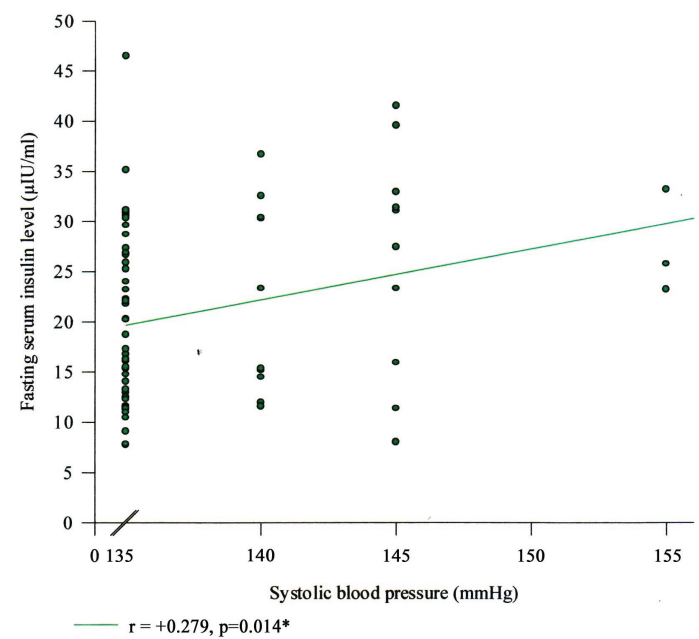

Fig.-1: Correlation between fasting serum insulin level and systolic blood pressure in study group $(n=50)$

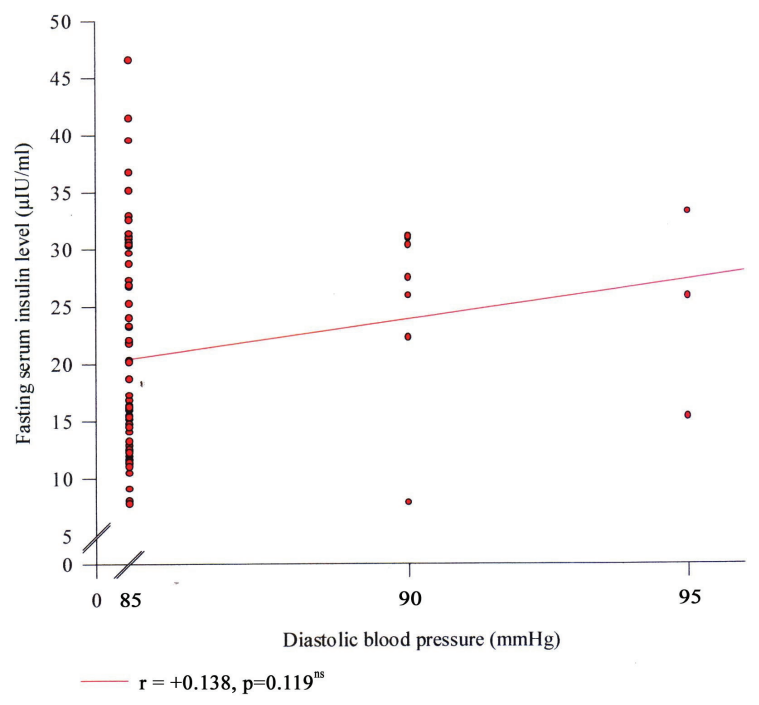

Fig.-2: Correlation between fasting serum insulin level and diastolic blood pressure in study group $(n=50)$ 


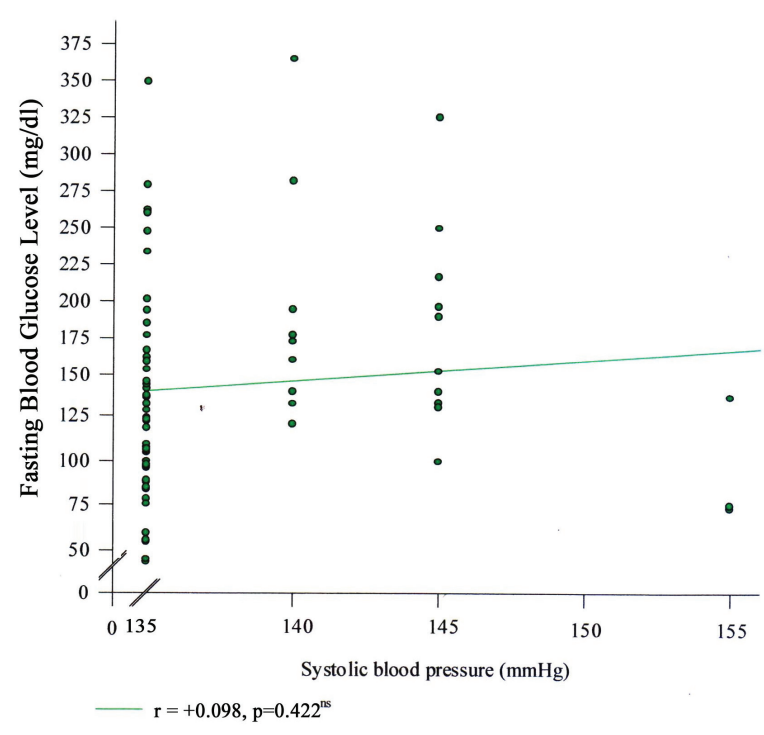

Fig.-3: Correlation between fasting blood glucose level and systolic blood pressure in study group $(n=50)$

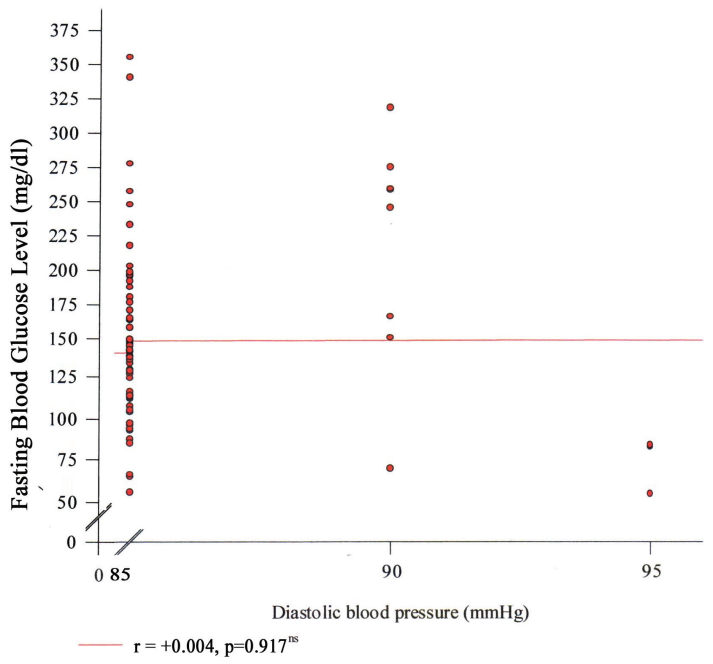

Fig.-4: Correlation between fasting blood glucose level and diastolic blood pressure in study group $(n=50)$

\section{Discussion}

In the present study, the levels of fasting blood glucose and fasting insulin in controls were almost within normal range and also similar to reported by the several investigators from abroad 6-9.

In this study, fasting insulin level in diabetic subjects was higher than that of controls and result was statistically significant. Similar types of observations were found by other workers. The fasting blood glucose level in diabetic subjects were also higher than those of controls and result was statistically significant.
Similar types of findings were reported by different researchers of different countries ${ }^{4-8}$. On the contrary, similar observations were made by other researcher but they did not find any significant difference in blood glucose level between the groups. ${ }^{21}$

Again, correlation analysis showed positive correlation of serum insulin level with systolic and diastolic blood pressure. In addition, blood glucose level also showed positive correlation with systolic and diastolic blood pressure. Similar observation was also reported by some investigators. ${ }^{22}$

Many explanations are suggested by different investigators regarding the development of hypertension in type 2 diabetic patient. It has been suggested that hyperinsulinemia and hyperglycemia which are the consequence of insulin resistance are associated with development of hypertension in type 2 diabetic patients. They suggested that hyperinsulinemia associated with increased sympathetic activity which leads to increase in circulating catecholamine. Increase in circulating catecholamine elevates the plasma renin activity. This high level of plasma renin elevates tissue angiotensin II. This angiotensin II is a potent vasoconstrictor and increases the total peripheral resistance, ultimately increase blood pressure. Again, angiotensin II acts directly on kidney and increase water, salt retention by the kidney and increase blood volume, increase cardiac output, increase blood pressure. 22,23 . In addition, hyperinsulinemia directly increase renal tubular reabsorption of sodium and also stimulates the synthesis of sodium retaining hormone, aldosterone and ultimately hypertension develops. Similarly, hyperinsulinemia with insulin resistance increased intracellular $\mathrm{Ca}++$ accumulation, increases secretion of growth factors and increases vascular smooth muscle cell proliferation, as a result narrowing of blood vessels and hypertension develops. 4,24. Again, some researcher suggested that chronic hyperglycemia is one of the important factor for development of oxidative stress. Oxidative stress plays a key role in pathogenesis of atherosclerosis and also causes endothelial dysfunction. Endothelial dysfunction associated with increase adhesion molecules, decrease endothelial-dependent vasodilation and nitric oxide (NO) production. All these are important factors for development of hypertension in type 2 diabetes mellitus. ${ }^{25}$ Moreover oxidative stress also stimulates the reninangiotensin-aldosterone system (RAAS) which is an important factor for development of hypertension in diabetic patients. ${ }^{26}$

In the present study, levels of fasting insulin and blood glucose are higher in diabetic subject than that 
of controls. Furthermore, in the present study, fasting insulin and blood glucose levels, showed positive correlation with systolic and diastolic blood pressure in diabetic adult female. These finding supports the relationship between insulin resistance and elevated blood pressure in diabetic adult female. But exact mechanism is not elucidated by this type of study due to time and financial constraints.

\section{Conclusion}

From this study, it can be concluded that hypertension, which is characterized by higher levels of systolic and diastolic pressure may present in diabetic adult female, may be due to insulin resistance.

\section{Acknowledgement}

The authors are thankful to the study subjects for their active, sincere and voluntary participation. The authors are also grateful to the Department of Physiology for all kind of support.

\section{References}

1 Ahmed.KA, Muniandy S, Ismail IS.Type 2 diabetes and vascular complication: A pathophysiological view. Biomed Res. 2010; 21 (2): 147-55

2. Guyton AC, Hall JE. Text Book of Medical Physiology. $11^{\text {th }}$ ed. SB Saunders, Mississipi.

3. Guariguata L, Whiting DR, Hambleton I. Global estimates of diabetes prevalence for 2013 and projections for 2035. Diabetes Res Clin Prect. 2013; 103(2014): 137-49.

4. Mercurio V, Carlomango G, Fazio V \& Fazio S . Insulin resistance is it time for primary prevention? World J Cardiol.2012; 4(1):1-7.

5. Pessin. JH \& Sal.Signatiel AR. Signaling pathways in insulin action: molecular targets of insulin resistance. J Clin Ivest.2000; 106(2):64-69.

6. Who. Hypertension control. Technical Report Series No 862,Geneva: 3020.

7. Kearney PM, Whelton. M,Reynolds. K, Whelton. PK. Global burden of hypertension: analysis of worldwide data. Lencet.2005; 365:217-223.

8. Theodire A. Kotchen.Obesity-related Hypertension?. Hypertension.2008; 52:801-802.

9. Cari de Luca \& Jerrold M, Olefsky. Inflammation and insulin resistance.NIH public Access. 2008; 582(1): 97-105.

10. Amanda N. Long, Do and Samuel Dagogo Jack. The comorbities of Diabetes and hypertension: Mechanism and Approach to target organ protection. J Clin Hypertens.2011;13(4):244-251.

11. Roberta Lima, Maarion Wofford and Jane F. Reckelhoff Hypertension in Postmenopausal women. Curr Hypertens Res.2012; 14(3): 254-260.
12. Soko Horita, Georage Seki, Hideoni Yaneda, Masashi Suzuki, Kazuhiko Koike and Toshiro Fujita.Insulin reistance induced hypertension. International journal of hypertension.2011;12(5):7-

13. Frank B Hu. MeirJ Stampfer.Insulin resistance and hypertension.Circulation.2005;112: 1678-1680.

14. Deepa R, Shanthi rani CS. Prevention of insulin resistance system in a selected south Indian population, Chenni. Indian J Med Res.2000;115: 198-27.

15. Chobanian AV, Bakris GL, Black HR, Cushan WC, Green LALzzo JL.The seventh Report of the Joint National Committee on Prevention, Detection, JNC 7 report. JAMA. 2003;289:2560-2572.

16. Garrison RJ, Kannel WB, Stokes J. Incidence and precursors of hypertension in young adults. The Framingham offspring study. Preventive medicine. 1987;16:235-

17 Sarzani R, Salvi F, Dessi- Fulgheri P, Rappelli A. Renin-angiotensin system, natriuretic peptides, obesity, metabolic syndrome and hypertension: an integrated view in human. J Hypertens. 2008; 26(5): 8 .

18. Frank,B, Meri.J, Stampfer.MD. Insulin Resistance and Hypertension. Circulation ;112:1687-1680.

19. Surapon Tangvarasiittichi. Oxidative stress, insulin resistance, dyslipidemia and type 2 diabetes mellitus.World journal of Diabetes 2015; 6(3):456480 .

20. Sulianwhitaker. Hypertension and insulin resistance. Circulation. 2008; 117(6)754-760.

21 . Koyong Park,Myron Gross,Duk-Hee Lee,Paul Holvoet, John H Himes, James M Shikany.Oxidative stress and Insulin Resistance. Diabetic Care.2009; 32(1): 1302-1307.

22. Salvetti A, Broji G, Dileje V, Bernini GP.The interrelationship between insulin resistance and hypertension. 1993;46(2):149-159.

23. Gerald M. Reavaen.Relationship Between Insulin resistance and Hypertension.Diabetic Care. 1991; 14(4):33-38.

24. Ele Ferrannini. Insulin and Blood pressure. Hypertension. 2005; 45(1):347-348.

25. Bernard M. Y.Cheung. Chao Li.Diabetes and Hypertension: Is There is a Common Metabolic pathway? Curr Atheroscler Rep.2012;14(1):160-166.

26. Tomaz J. Guzik, Shafi Mussa, MA,Daniela Gastaldi Gastaldi, MD, Jerzi Sadowski,Chandi Ratnatunga, Ravi Pillai, Keith M. Channon. Mechanisms of increased vascular superoxide production in human diabetes mellitus.Circulation.2002; 105:1656-1662. 